\title{
Evaluating the Accuracy of an Equivalent Linear Model in Predicting Peak Displacement of Seismic Isolation Systems using Single Friction Pendulum Bearings
}

\author{
Thanh-Truc Nguyen", Nhan Dinh Dao ${ }^{2 *}$ \\ ${ }^{1}$ Civil Engineering Faculty, Mien Tay Construction University, 20B Pho Co Dieu, Vinh Long City, Vinh Long, Vietnam \\ ${ }^{2}$ Civil Engineering Faculty, University of Architecture Ho Chi Minh City, 196 Pasteur, Dist. 3, Ho Chi Minh City, Vietnam \\ ${ }^{*}$ Corresponding author, e-mail: nhan.daodinh@uah.edu.vn
}

Received: 13 December 2021, Accepted: 07 September 2021, Published online: 17 September 2021

\begin{abstract}
This study evaluates the accuracy of an equivalent linear model in predicting peak nonlinear time-history displacement of seismic isolation systems with single friction pendulum bearings. To perform this evaluation, dynamic response of numerical models of 120 isolation systems subjected to 390 strong earthquake ground motions, including motions with pulse and motions without pulse, was analyzed and statistically processed. The results show that the equivalent linear model can partly predict the peak displacement of its counterpart nonlinear model. However, the equivalent model can also underestimate or overestimate the peak displacement. On average sense, the equivalent linear model underestimates small peak displacement and overestimates large peak displacement. It is also observed that the relationship between linear and nonlinear peak displacements depends on ground motion types. Based on the analysis data, equations representing relationship between linear and nonlinear peak displacements at different reliable levels for different ground motion types were proposed. These equations can be used in practice.
\end{abstract}

\section{Keywords}

equivalent linear model, isolation system, earthquake engineering, time history nonlinear analysis, displacement demand

\section{Introduction}

Base isolation system (BIS) is an effective method to mitigate damage of buildings during earthquakes. A BIS includes flexible devices, called seismic isolators or bearings, installed beneath a building to "isolate" it from the ground. A seismic isolator is stiff in vertical direction to support vertical load with limited vertical deformation but flexible in horizontal direction to provide flexibility to the isolated building in this direction. Fig. 1 depicts two common types of seismic isolator. Fig. 1(a) shows the composition of a lead rubber bearing and Fig. 1(b) presents a concave friction bearing. Readers may refer to [1] for more detail about these bearing types.

The flexibility provided by seismic isolators lengthens natural period of the isolated building thus reduce its base shear coefficient as demonstrated in Fig. 2(a). The flexibility also increases the base displacement of the isolated building as shown in Fig. 2(b). A large base displacement increases the clearance between the isolated building and surrounding structures. It also introduces large overturning moment to both bearings and foundation system.

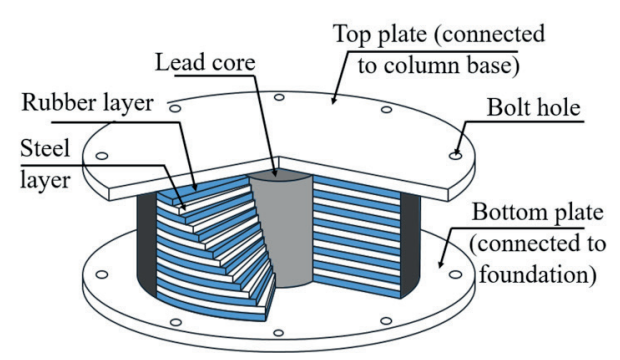

(a)

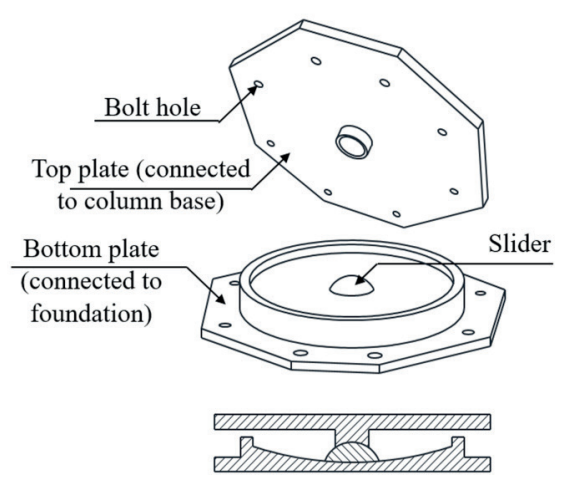

(b)

Fig. 1 Seismic isolators a) Lead rubber bearing, b) Friction pendulum bearing 


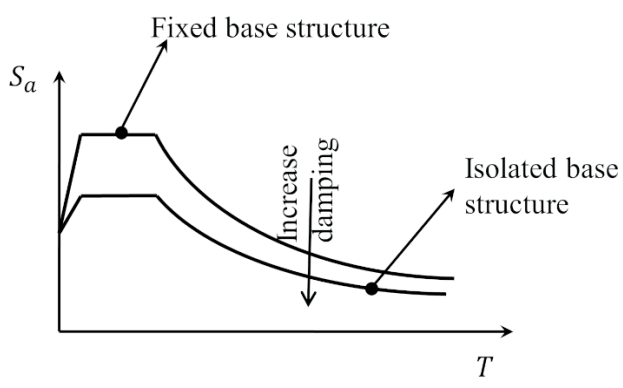

(a)



(b)

Fig. 2 Typical design spectra a) Spectral acceleration, b) Spectral displacement

Because of this, accurately predicting peak displacement of isolation system, which equals the base displacement, is an important task in designing isolated buildings.

Although peak displacement of an isolation system can be estimated through a time-history nonlinear analysis procedure, many contemporary design codes $[2,3]$ allow using an equivalent linear approach to analyze isolated buildings, including analyzing peak displacement of the isolation system, providing that they are not irregular.

Equivalent linear approaches are computational efficient, easy to perform and provide more intuition to practical engineers. Furthermore, many practical design equations have been derived base on linear system assumption. This fact calls for not only a good understanding of equivalent linear approaches but also their accuracy.

An equivalent linear approach replaces the nonlinear hysteresis behavior of a seismic isolator by an "equivalent" linear behavior represented by linear stiffness and viscous damping. Most approaches are based on either stationary response or steady-state harmonic response of the counterpart nonlinear system [4]. Stationary response approach, which is useful for calculating statistical parameters of the response given the statistical parameters of the input, determines equivalent linear stiffness and viscous damping to minimize the mean-squared error between the response of the nonlinear system and the response of the linearized system [5]. Steady-state harmonic response approach determines equivalent linear stiffness and viscous damping based on a secant stiffness, usually at peak displacement, of the nonlinear system. A study by Rosenblueth and Herrera [6] showed that steady state displacement of a slightly nonlinear system subjected to harmonic loading is similar to that of a linear viscoelastic system whose stiffness equals the secant stiffness of the nonlinear system at peak displacement and damping ratio is determined such that energy dissipated in a cycle between the two systems are equal. This result becomes the background for the equivalent linear procedure currently in use $[2,3]$.

It should be noticed, however, that all linear approaches are approximate and the secant stiffness approach was developed for steady state response of undamped slightly nonlinear systems subjected to harmonic loadings whereas the response of a nonlinear system to an earthquake motion is not steady and its peak displacement response usually occurs at only one instant. Therefore, the accuracy of the secant stiffness approach, as well as other linearization approaches, in predicting peak displacement of isolation systems was the objective of many past studies. However, the conclusions were conflicting. A study by Franchin et al. [7] investigated the accuracy of three equivalent linear models on three isolated bridge models and concluded that the accuracy of the linearized models is unpredictable and the use of linearized models can lead to an error of more than $100 \%$, compared to nonlinear results. Dicleli and Buddaram [8] observed that equivalent linear models generally underestimate peak displacement of isolation system. Additionally, the accuracy of the model depends on many parameters, including ground motions characteristic and mechanical properties of the isolation system. Mavronicola and Komodromos [9] carried out a case study on 3-story and 5-story isolated building models subjected to six ground motions and concluded that equivalent linear models conservatively predict peak displacement of isolation systems. Jara et al. [10] evaluated the accuracy of an equivalent linear model with improved damping ratio in predicting peak displacement of 120 isolated bridge models subjected to 26 ground motions and concluded that the equivalent linear model is in good agreement with the nonlinear models in predicting peak displacement of isolation system. Liu et al. [11] performed an extensive evaluation on the accuracy of equivalent linear models in predicting peak displacement isolated SDOF systems. The study concluded that the accuracy depends on many assumptions and there is no best equivalent linear model that best predicts peak displacement of all systems. Jara et al. [12] investigated the accuracy of 
equivalent linear models in predicting nonlinear displacement of three types of irregular isolated bridge models and found that an equivalent linear model can under- or over-estimate the peak displacement and their accuracy depends on ductility demand. Dao et al. [13] evaluated the effect of ground motion types and mechanical properties of isolation system on the accuracy of an equivalent linear procedure employing Rosenblueth and Herrera [6] linearized approach, which is used in this study, in predicting peak displacement of isolation systems. The study proposed equations for predicting the accuracy of the predicted peak displacement as functions of isolation system's property and ground motion type. Beside these researches on isolation systems, there have been also many studies on the accuracy, limitation and improvement of the secant stiffness approach on other nonlinear systems [14-20].

Most researches evaluated the accuracy of equivalent linear models on isolated structures using lead rubber bearings, whose behavior contains initial elastic response. Very few studies have ever investigated friction isolation systems, which possess abrupt change in stiffness when sliding starts. Fadi and Constantinou [21] investigated the accuracy of an equivalent linear procedure which employs Rosenblueth and Herrera [6] linearized approach on nine triple friction pendulum systems subjected to two sets of ground motions. The study showed that the linear procedure provides good and often conservative estimation of peak displacement of the isolation systems. The study by Dao et al. [13] mentioned earlier can extent to include friction pendulum systems, but it does not account for the velocity-dependent effect of friction coefficient between sliding surfaces in the system. Both [13] and [21] examined an equivalent linear procedure employing an equivalent linear model but did not directly examine the equivalent model for every single ground motions.

To the best of our knowledge, there has not been any study that ever explicitly evaluated the accuracy of equivalent linear models in predicting peak displacement of isolation systems using friction pendulum bearings and propose equations to modify the predicted peak displacement. This study aims to numerically investigate the accuracy of an equivalent linear model on a large number of friction isolation systems using single friction pendulum bearings subjected to different types of earthquake ground motions. Based on the numerical data, equations representing the relationship between peak linear displacement, which is computed from the analysis of the equivalent linear model, and peak nonlinear displacement, which is obtained from the analysis of a nonlinear model, at different probabilities will be proposed. The equations can be used to estimate the peak nonlinear displacement at a certain reliability given a linear peak displacement.

To obtain these objectives, the next section presents modeling assumption and nonlinear model of isolation systems using single friction pendulum bearings for investigation. The equivalent linear model and analysis procedure are also presented in the next section. Input earthquake ground motions for dynamic time-history analysis are addressed in Section 3. Section 4 discusses the numerical results and proposed equations representing the relationship between linear and nonlinear peak displacements of the isolation systems at different reliabilities. The final section summarizes this research and recommends further possible studies to improve the numerical results.

\section{Theoretical backgrounds}

This study investigates isolation systems using single friction pendulum bearings, as mentioned before. For simplicity, superstructures were considered to be rigid, given that it is much stiffer than their isolation system. This assumption was widely used in past researches [8, 9, 12, 13, 21]. To develop the hysteresis behavior of the bearing, consider a rigid mass supported by a single friction pendulum bearing which can be idealized as a system shown in Fig. 3. Forces acting on the mass include weight $W$, normal reaction $f_{n}$, tangent force (friction force) $f_{t}$, and horizontal force acting on the bearing $V$. Applying small displacement assumption, the relationship between horizontal displacement $u$ and horizontal force $V$ of the system can be written as Eq. (1) [22].

$V=\mu W \operatorname{sign}(\dot{u})+(W / R) u$,

where $R$ and $\mu$ are radius and friction coefficient of the sliding surface.

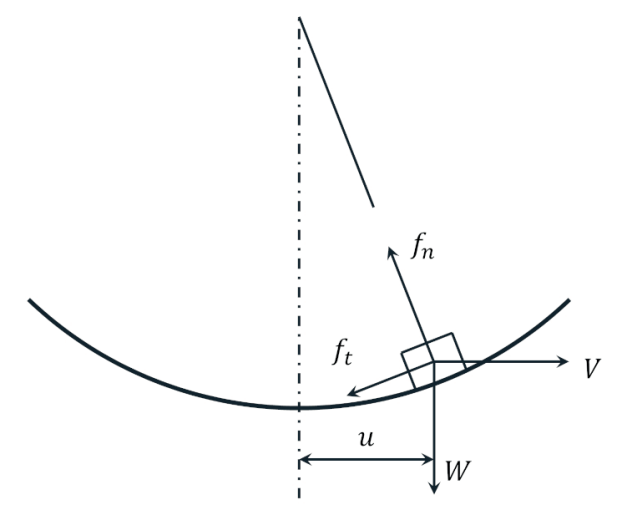

Fig. 3 Single friction pendulum bearing model 
Eq. (1) shows the force - displacement relationship of a single friction pendulum bearing at a displacement $u$ and sliding velocity $\dot{u} \cdot \operatorname{sign}(\dot{u})$ is a signum function on $\dot{u}$, which returns $+1,-1$ and 0 if $\dot{u}>0, \dot{u}<0$ and $\dot{u}=0$, respectively. The relationship between $u$ and $V$ in a symmetric cycle with maximum displacement $u_{m}$ is shown in Fig. 4. This is the well-known hysteresis loop of a single friction pendulum bearing.

When a system shown in Fig. 3 is subjected to a ground motion $u_{g}$, the governing differential equation of motion can be written as [22]:

$$
\ddot{u}+(c / m) \dot{u}+\mu \operatorname{gsign}(\dot{u})+(g / R) u=-\ddot{u}_{g} .
$$

In this equation, $g$ is the gravity acceleration and $c$ is viscous damping coefficient representing the energy dissipation of the system. For simplification, $c$ is represented through a damping ratio $\zeta$ as following:

$c=2 \zeta m \omega_{p}$,

where pendulum angular frequency $\omega_{p}$ is computed by:

$\omega_{p}=\sqrt{R / g}$.

Substituting Eqs. (3) and (4) into Eq. (2) yields:

$\ddot{u}+2 \zeta \sqrt{R / g} \dot{u}+\mu g \operatorname{sign}(\dot{u})+(g / R) u=-\ddot{u}_{g}$.

Eq. (5) indicates that the displacement response $u$ of an isolated mass is independent on $m$. Thus any value of $m$ can be used for analyzing displacement response.

It should be noted that for a self-lubricating single friction pendulum bearing, which composes of a slider coated with polytetrafluoroethylene (PTFE) sliding on a concave stainless-steel surface (Fig. 1(b)), the friction coefficient $\mu$ between sliding surfaces is not a constant but is a function of many parameters such as sliding velocity, contact

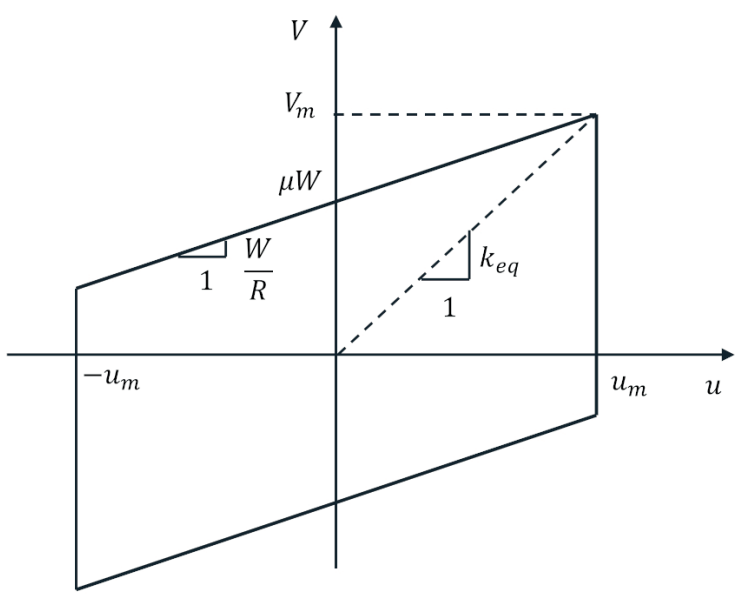

Fig. 4 Hysteresis loop of single friction pendulum bearings pressure and temperature [23-25]. The dependency of friction coefficient on these parameters is demonstrated in Fig. 5. Among them, sliding velocity appears to have the most influence when the system is subjected to earthquake ground motions. Because of that, many researchers have employed a velocity-dependent friction coefficient model for their studies [26-29]. The most widely used model is [30]:

$\mu=\mu_{\text {fast }}-\left(\mu_{\text {fast }}-\mu_{\text {slow }}\right) e^{-r v}$,

where $\mu_{\text {fast }}$ and $\mu_{\text {slow }}$ are respectively friction coefficients at fast and slow sliding velocities, $v$ is sliding velocity between surfaces and $r$ is a rate parameter. In this model, friction coefficient $\mu$ increases with the increasing of sliding velocity $\nu$. Specifically, $\mu$ increases from $\mu_{\text {slow }}$ at a very low sliding velocity to $\mu_{\text {fast }}$ at a high velocity (usually at around $25 \mathrm{~cm} / \mathrm{s}$ ). Beyond this high value, velocity has little effect on friction coefficient.

Friction coefficients $\mu_{\text {slow }}$ and $\mu_{\text {fast }}$ depends on applied axial load on the bearing and its load capacity. Cardone et al. [28] observed from experimental data that $\mu_{\text {fass }} / \mu_{\text {slow }}$ for usual bearings ranges from 2.0 to 3.2. The study adopted $\mu_{\text {fast }} / \mu_{\text {slow }}=2.5$ in its investigation.

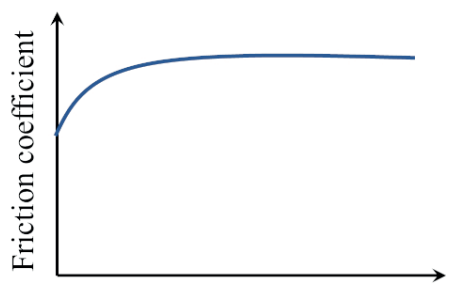

Sliding velocity
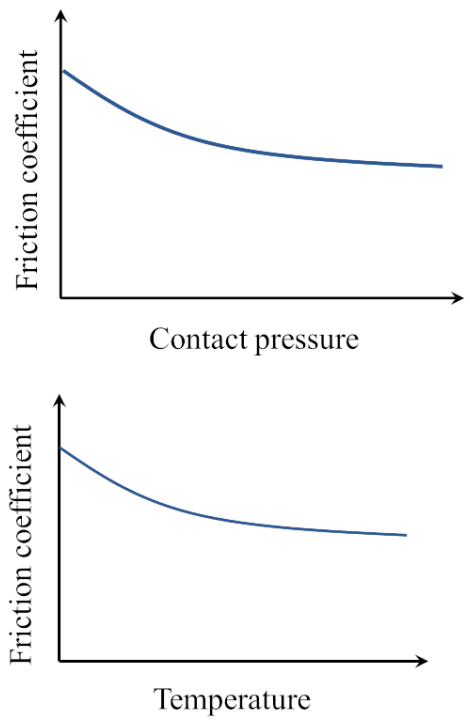

Fig. 5 Dependency of friction coefficient on velocity, contact pressure and temperature 
Rate parameter $r$ is a function of air temperature and contact pressure between sliding surfaces [23-25, 30] and can be evaluated by assuming a reference sliding velocity $v_{\text {ref }}$ and the correspondent reference friction coefficient $\mu_{\text {ref }}$ Cardone et al. [28] assumed that $v_{r e f}=200 \mathrm{~mm} / \mathrm{s}$ and $\mu_{r e f}=0.8 \mu_{\text {fast }}$. Accordingly, $r$ can be determined by Eq. (7).

$r=\frac{1}{v_{\text {ref }}} \ln \frac{\mu_{\text {fast }}-\mu_{\text {slow }}}{\mu_{\text {fast }}-\mu_{\text {ref }}}=0.0055(\mathrm{~s} / \mathrm{mm})$

Eqs. (6-7) constitute a friction coefficient model for single friction pendulum bearings subjected to earthquake motions, whose differential governing equation is presented by Eq. (2). Displacement response of the isolation system, $u$, in that equation can be solved using numerical methods. However, solving that equation for a large number of earthquake motions is time consuming. Thus, many design codes allow using equivalent linear procedures, which based on equivalent linear models, to predict peak displacement of isolation systems. The most-used equivalent linear model is based on a secant stiffness at steady harmonic amplitude proposed by Rosenblueth and Herrera [6] and an equivalent viscous damping model proposed by Jacobsen [31]. The equivalent linear parameters of the model are presented next.

Eq. (2) is the governing differential equation of motion for a single degree of freedom system having nonlinear hysteretic behavior shown in Fig. 4. The governing equation of its equivalent linear model can be expressed as:

$\ddot{u}+\left(c_{e q} / m\right) \dot{u}+\left(k_{e q} / m\right) u=-\ddot{u}_{g}$,

where $k_{e q}$ and $c_{e q}$ are respectively equivalent linear stiffness and equivalent linear viscous damping coefficient evaluated at the peak displacement $u_{m}$ of the system.

From Fig. $4, k_{e q}$ can be computed as:

$k_{e q}=\mu W / u_{m}+W / R$.

The equivalent $c_{e q}$ is computed such that the energy dissipated in one complete cycle of the equivalent linear system is as the same as that of the nonlinear system. Neglecting viscous damping in the nonlinear system, the energy dissipated $E_{H}$ in one cycle at displacement $u_{m}$ equals the area of the hysteretic loop at the correspondent displacement (Fig. 4). Accordingly, $E_{H}$ is computed by:

$E_{H}=4 \mu W u_{m}$.

The energy dissipated in one cycle of steady state oscillation of the equivalent linear system, $E_{S}$, is computed by [32]:
$E_{S}=\pi c_{e q} \omega_{n} u_{m}^{2}$,

where $\omega_{n}$ is natural angular frequency of the equivalent linear system, which can be computed from Eq. (12).

$\omega_{n}=\sqrt{k_{e q} / m}$

Equating $E_{H}$ from Eq. (10) and $E_{S}$ from Eq. (11) yields:

$c_{e q}=4 \mu W / \pi \omega_{n} u_{m}$.

If viscous damping of the nonlinear system is included, then the viscous damping coefficient of the equivalent linear system is computed by:

$c_{e q}=4 \mu W / \pi \omega_{n} u_{m}+2 \zeta m \omega_{p}$.

The last term in the above equation is the viscous damping coefficient of the nonlinear system computed by Eq. (3).

Note that the hysteresis loop in Fig. 4 is for a constant friction coefficient model. However, friction coefficient in friction bearings is velocity-dependent as presented earlier. Thus an equivalent friction coefficient $\mu_{e q}$ shall be used to determine $k_{e q}$ and $c_{e q}$. According to Nhan and Ai [33], a constant friction coefficient of $\mu_{e q}=0.88 \mu_{\text {fast }}$ best predicts peak displacement of isolation systems using single friction pendulum bearings. This value shall be used in this investigation.

Both equivalent stiffness $k_{e q}$ and equivalent viscous damping coefficient $c_{e q}$ computed from Eqs. (9) and (13), respectively, are dependent on peak displacement $u_{m}$, which in turn depends on $k_{e q}$ and $c_{e q}$ as shown in Eq. (8). Thus $u_{m}$ can only be obtained through an iteration process. The procedure for computing $u_{m}$ of the equivalent linear model is as following:

Step 1: Select a trial peak displacement $u_{m, o l d}$

Step 2: Compute the effective stiffness $k_{e q}$ and effective damping coefficient $c_{e q}$ corresponding to $u_{m, o l d}$ using Eqs. (9) and (13).

Step 3: Perform time history analysis of the linear model with $k_{e q}$ and $c_{e q}$ (following Eq. (8)) then obtain the new peak displacement $u_{\text {m,new }}$

Step 4: Assign the $u_{m, \text { new }}$ to the $u_{m \text {,old }}$ and redo Steps 2 and 3 until the $u_{m \text {,new }}$ and the $u_{m, o l d}$ converge. The tolerance in this study was $0.1 \mathrm{~mm}$.

In this study, the governing differential equation of the nonlinear system (i.e., Eq. (2)) and the governing differential equation of the linear system (Eq. (8)) were solved through computational models built in OpenSees software [34]. Furthermore, the computational models were 
extended to 3-D single mass models to consider two horizontal components of earthquake ground motions. In the nonlinear model, single friction pendulum bearing was modeled by a Triple Friction Pendulum element [35], which can be used to model 3-D single, double and triple friction pendulum bearings. The velocity-dependent friction coefficient model represented by Eq. (6) was employed. Equivalent friction coefficient $\mu_{e q}$ ranging from 0.02 to 0.16 with an incremental of 0.01 and pendulum period $T_{p}$ ranging from $2.0 \mathrm{~s}$ to $5.5 \mathrm{~s}$ with an incremental of $0.5 \mathrm{~s}$ were investigated. This resulted in 15 friction coefficient values and 8 pendulum period values, which yielded a total of 120 isolation system models to be investigated. These wide ranges of bearing's parameters are expected to cover most practical applications.

\section{Input ground motions}

Three-hundred-and-ninety records of strong ground motions considered in GCR 11-917-15 project [36] were used for numerical investigation. These ground motion data were recorded from earthquake events with magnitude stronger than 6.0 and closest source-to-site distance not greater than $30 \mathrm{~km}$. Eighty-eight ground motions among the selected records were classified as pulse-like motions. To investigate the effect of ground motion type on the accuracy of the equivalent linear model, the selected ground motions were classified into 'Without Pulse' (NP) motion group and 'With Pulse' (WP) motion group. Mean spectral acceleration, i.e. the square root of sum squared of the two components of a ground motion pair, of these two groups are presented in Fig. 6. The mean spectral acceleration of NP motions is plotted together with the average mean spectral acceleration over all NP motions in Fig. 6(a). Figs. 6(b) displays similar information for WP motions. These figures show that, on average sense, spectral acceleration of WP motions is much stronger than that of NP motions, especially at long period range, which matches the effective period of most isolation systems.

Because the number of the selected records (390) is large, it is not convenient to list them here. Readers may refer to Tables C1-2 of [36] for detail information of the selected ground motions.

\section{Numerical results}

Fig. 7 shows the responses of the nonlinear model of an isolation system with $\mu_{e q}=0.06, T_{p}=3.5 \mathrm{~s}$ and its equivalent linear system subjected to Takatori motion recorded from Kobe 1995 earthquake. The equivalent linear system

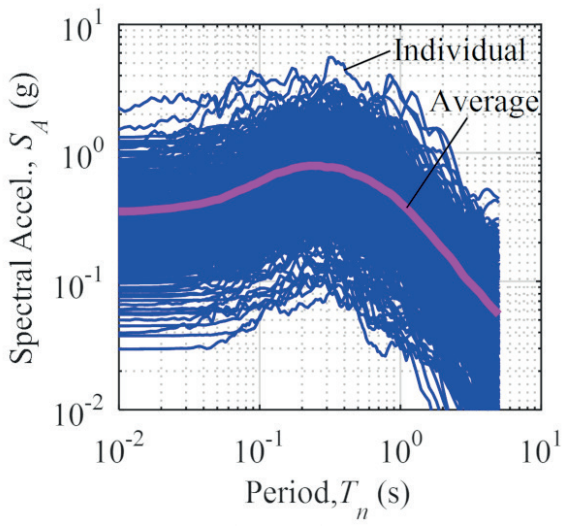

(a)

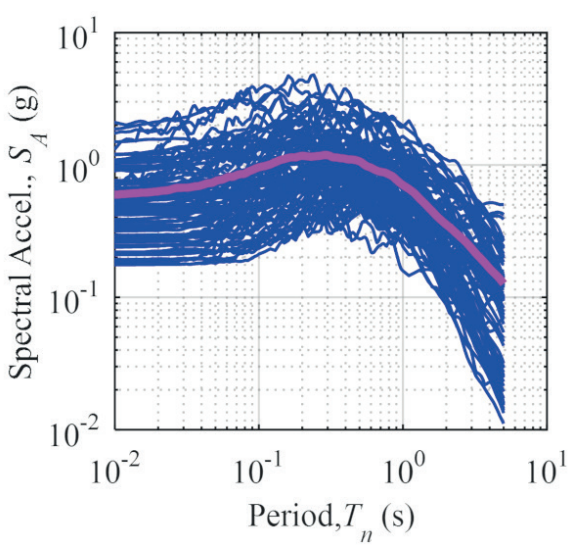

(b)

Fig. 6 Spectral acceleration of selected ground motions

was the system at the converged state of the procedure for computing $u_{m}$ described in Section 2. Figs. 7(a), (b) show the ground motion components in the $\mathrm{X}$ - and $\mathrm{Y}$ - direction, respectively. The displacement trace of the two models is shown in Fig. 7(c). The peak displacement of the nonlinear model is $0.477 \mathrm{~m}$, which is marked by a plus sign in the figure, and the peak displacement of the linear model is $0.556 \mathrm{~m}$. The dash line in this figure represents the direction at which the peak displacement of the nonlinear model occurs, which is hereafter referred to as "peak displacement direction". Fig. 7(d) shows normalized hysteresis loop of the two models in the peak displacement direction. The horizontal axis represents displacement $u$ in the peak displacement direction while the vertical axis represents normalized force $f$ in the same direction. The normalized force in a model is computed by dividing total horizontal force by vertical force of that model.

The result from Fig. 7 indicates that the response of the linear model is evidently different from that of the counterpart nonlinear model. The peak displacement of the linear model exceeds the peak displacement of the nonlinear model by $16.6 \%$. However, this amount of exceedance 
does not always occur. For a certain nonlinear model, the equivalent linear model overestimates peak displacement for a motion but underestimates it for another motion.

Fig. 8(a) shows relationship between linear peak displacement $u_{l}$ and nonlinear peak displacement $u_{n}$. This figure was constructed from 15,633 data points with $u_{l}$ ranging from $0.05 \mathrm{~m}$ to $1.00 \mathrm{~m}$. For practical meaningfulness, only data points with $u_{l}$ smaller than 0.3 times

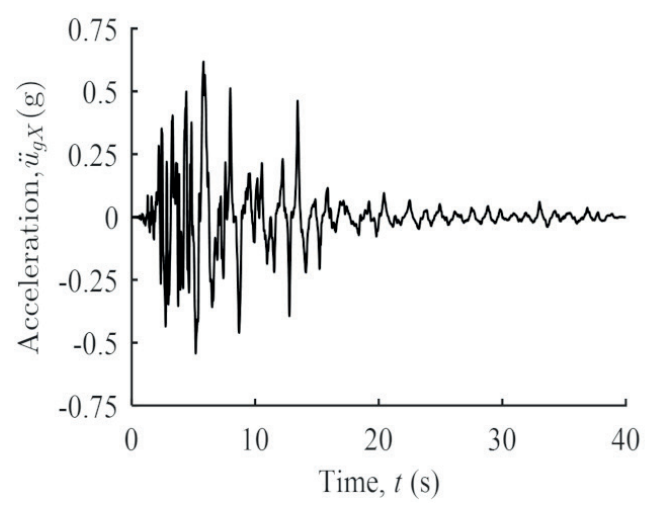

(a)

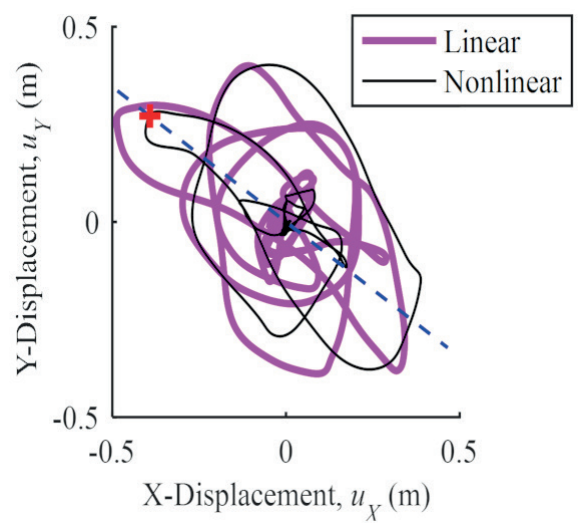

(c) the pendulum radius were selected [37]. The data points were obtained from the analysis of 120 models (correspondent to 15 friction coefficients and 8 pendulum periods as described in Section 2) subjected to 390 selected ground motions described in Section 3. The dash line in the figure representing the data points where $u_{n}$ equals $u_{l}$, i.e., when an equivalent linear model precisely predicts the peak displacement of its counterpart nonlinear model.

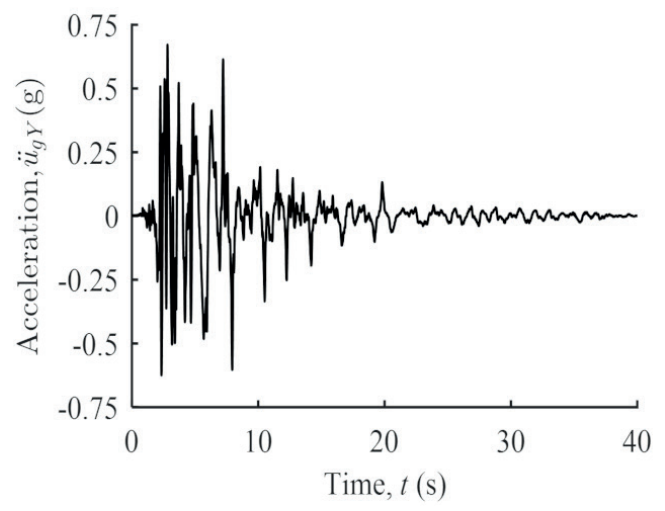

(b)

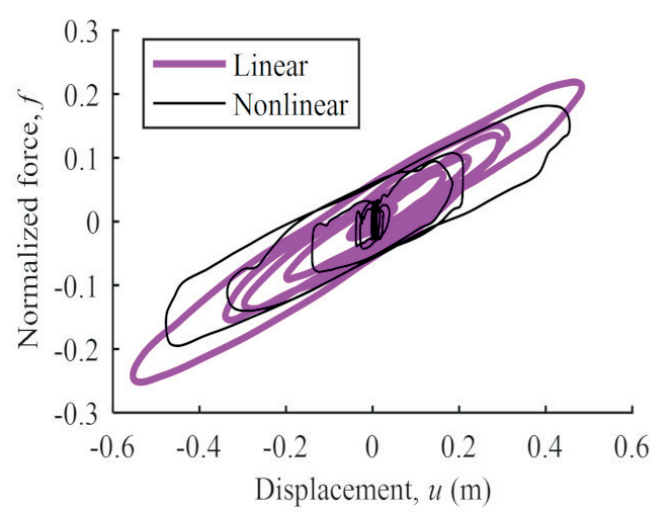

(d)

Fig. 7 Input and response of an isolation system to Takatori motion a) Input acceleration in the X-direction, b) Input acceleration in the Y-direction, c) Dicplacement trace, d) Hysteresis loop in the peak displacement direction

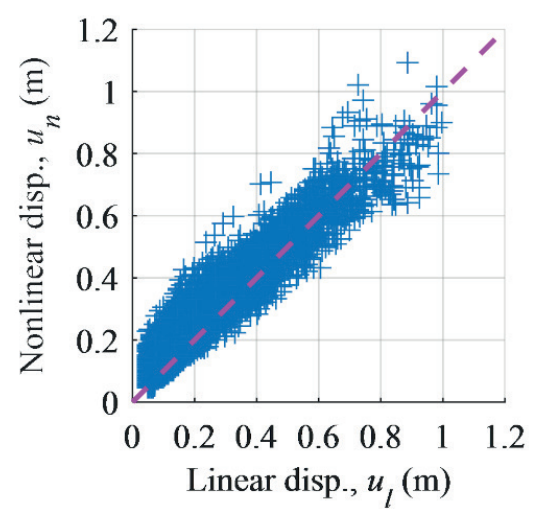

(a)

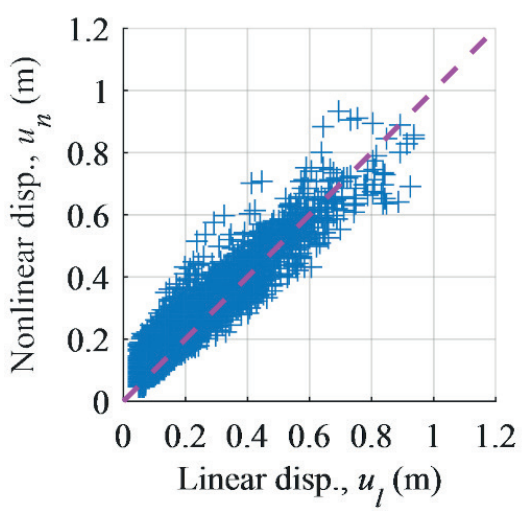

(b)

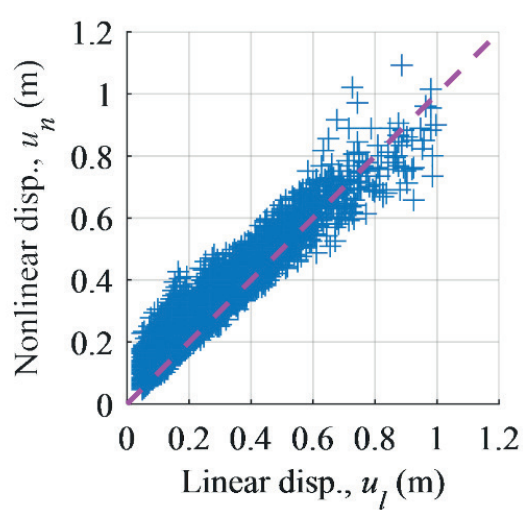

(c)

Fig. 8 Relationship between peak linear and peak nonlinear displacement a) All motions, b) Motions without pulse, c) Motions with pulse 
The figure shows that the data points generally gather along the dash line, which implies that an equivalent linear model can partly predict the peak displacement of its counterpart nonlinear model. However, because the equivalent linear model is approximate, there is dispersion of the data points along the line and equivalent linear model may underestimate or overestimate the peak displacement. At small peak displacement, there are more data points above the dash line than below the dash line. This trend reverses at large displacement. This means that, on average sense, an equivalent linear model underestimates peak displacement at small values and overestimates it at large values.

The data points from Fig. 8(a) were classified according to ground motion types as shown in Figs. 8(b) and (c). It can be observed from these figures that the selected NP ground motions did not drive the investigated isolation systems to a very large displacement while the selected WP motions generated lots of data points at large peak displacement. This agrees with the results in Fig. 6 that the average spectral acceleration of the selected WP motions is much stronger than that of the selected NP ground motions.

To develop the relationship between the peak linear displacement $u_{l}$ and the peak nonlinear displacement $u_{n}$, the data presented in Fig. 8 were statistically processed. Accordingly, the data points were grouped into bins base on $u_{l}$. Specifically, there are 14 bins with equal space of $u_{l}$ between $0.05 \mathrm{~m}$ to $0.4 \mathrm{~m}$ and 7 bins equally spaced between $0.4 \mathrm{~m}$ to $1.0 \mathrm{~m}$. Only data bins that contain at least 20 data points were statistically processed with the assumption that $u_{n}$ in each bin follows a normal distribution. Fig. 9 shows the relationship between the mean-value as well as $90 \%$-of-exceedance-value of $u_{n}$, respectively denoted by $u_{n 50}$ and $u_{n 90}$, and the mean value of $u_{l}$ for every bin. The figure indicates that the relationships between $u_{n 50}$ as well

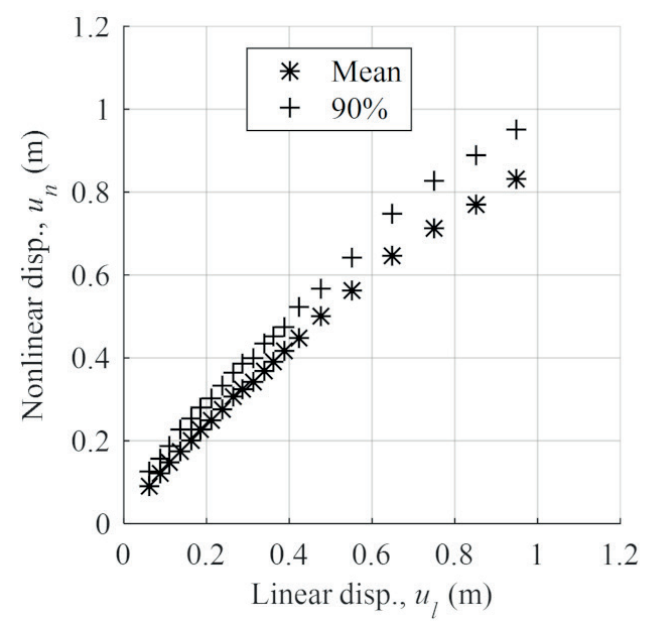

Fig. 9 Relationship between linear and nonlinear displacements as $u_{n 90}$ and $u_{l}$ are nonlinear. It can be observed that, on average sense, the equivalent linear model underestimates peak displacement at small displacement and overestimates it at large displacement, as commented before.

For practical application, the fitted curves representing relationship between $u_{l}$ and $u_{n}$ at different probabilities of exceedance shall be developed. This study assumes that the relationship follows a polynomial function. To select the order of the fitted polynomial functions, three function types, which are linear, parabolic, and cubic functions, were tested.

The selected data, which contained 15,633 samples, were randomly grouped into a training set and a testing set. The training set took 10,943 samples, which is approximately $70 \%$ of the full data, and the testing set took the remaining samples. The training set was used to develop fitted functions whereas the testing set was used to test the validity of the fitted functions.

The training set was grouped into 14 bins, which were then statistically processed to find their mean $u_{l}$ and $u_{n 50}$ as described earlier. A similar process was performed on the testing set. The relationship between mean $u_{l}$ and $u_{n 50}$ of the training set was then fitted by linear, parabolic, and cubic functions. The coefficient of determinacies $R^{2}$ of the fitted functions were calculated for both the training set and the testing set.

Because the samples in the training and testing sets were randomly selected, the fitted functions and their $R^{2}$ can vary from trial to trial. To provide a good judgement, 20 trials following the above process were performed. The $R^{2}$ for both training and testing sets of these trials are plotted in Fig. 10. The fitted functions, which are different between trials, are not presented here for brevity.

As expected, for the training sets (Fig. 10(a)), the $R^{2}$ is smallest for linear fitting and largest for cubic fitting in all trials. The $R^{2}$ of the parabolic fitting is close to that of the cubic fitting. For the testing sets (Fig. 10(b)), the $R^{2}$ of the parabolic fitting is either close to or greater than the $R^{2}$ of the cubic fitting. This result indicates that using cubic (or higher order) fitting may encounter a high variance fitting problem. Because of this, parabolic fitting shall be used to fit the relationships between $u_{l}$ and $u_{n 50}$, as well as $u_{n 90}$ for consistency.

The best fit parabolic curves passing through the data points in Fig. 9 are:

$$
\begin{aligned}
& u_{n 50}=-0.3011 u_{l}^{2}+1.142 u_{l}+0.02139, \\
& u_{n 90}=-0.29 u_{l}^{2}+1.28 u_{l}+0.05404 .
\end{aligned}
$$




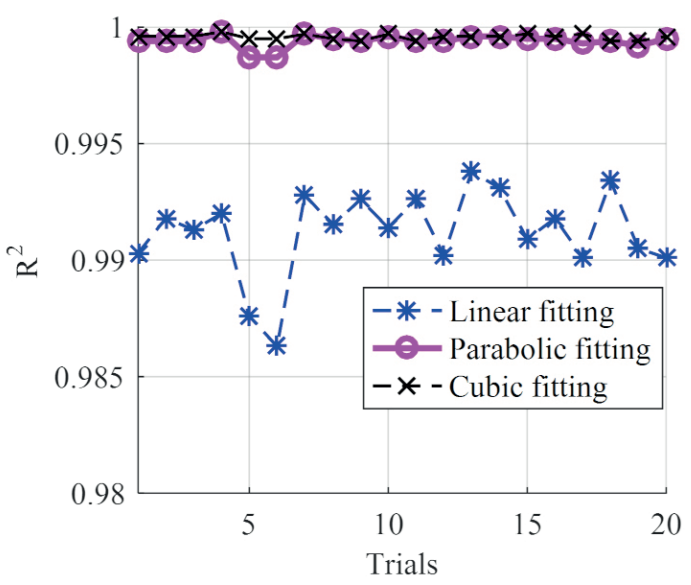

(a)

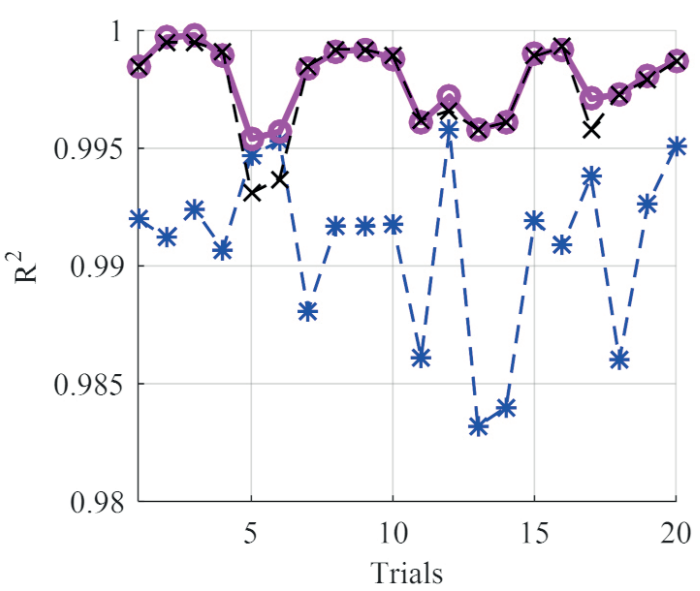

(b)

Fig. 10 Coefficient of determinacies for training and testing sets a) Training sets, b) Testing sets

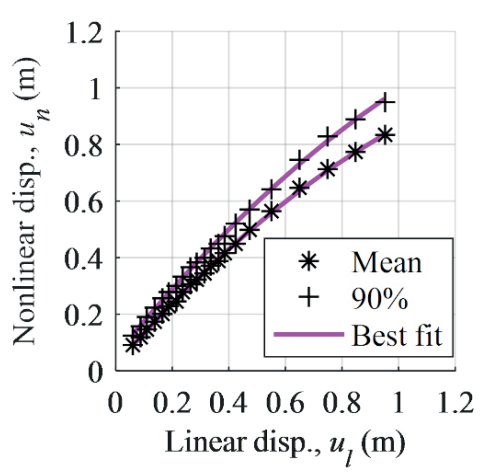

(a)

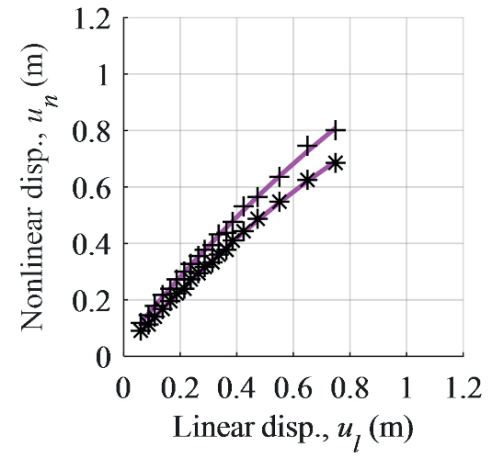

(b)

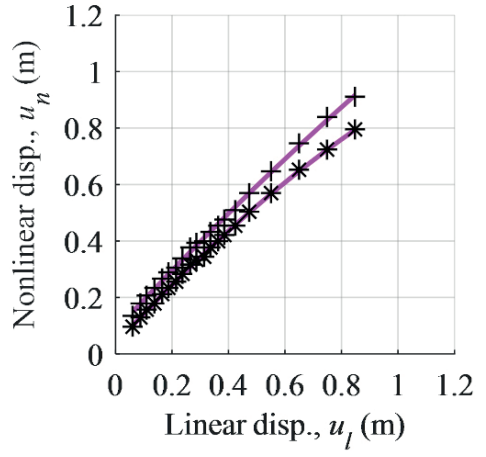

(c)

Fig. 11 Best fit curves at different probability of exceedance a) All motions, b) Motions without pulse, c) Motions with pulse

The coefficient of determination for these fitted equations are $R^{2}=0.99984$ and $R^{2}=0.99943$ for $u_{n 50}$ and $u_{n 90}$, respectively. Note that the data presented in Fig. 9 are for all motions data, which contains 15,633 samples. The fitted curves are plotted along with the data points in Fig. 11(a).

The best fitted curves for data points of NP ground motions, and WP ground motions were also developed. These curves are plotted in Figs. 11(b) and (c). The equations of these curves and their R-squared values are presented in Table 1.

For visual comparison, the fitted curves for all ground motions sets are plotted together in Fig. 12. The figure shows that the fitted equation developed from WP ground motion set predicts largest $u_{n}$ at a certain $u_{l}$, both at mean value and 90\%-probability-of-exceedance value. The equation derived from all ground motion set generates a slightly smaller $u_{n}$. For application, the equation that fits all ground motion set, i.e., Eqs (15-16), should be used because it covers all ground motion types and does not much underestimate the equation developed from WP ground motion set.
Table 1 Caption to Table (Style name: PP Figure/Table caption)

\begin{tabular}{lc}
\hline Equation & R-squared \\
\hline Motions without pulse (NP motions) & \\
$u_{n 50}=-0.2864 u_{l}^{2}+1.109 u_{l}+0.02090$ & 0.99977 \\
$u_{n 90}=-0.2897 u_{l}^{2}+1.2361 u_{l}+0.04568$ & 0.99897 \\
Motions with pulse (WP motions) & \\
$u_{n 50}=-0.2401 u_{l}^{2}+1.100 u_{l}+0.03438$ & 0.99977 \\
$u_{n 90}=-0.1181 u_{l}^{2}+1.0832 u_{l}+0.08320$ & 0.99927 \\
\hline
\end{tabular}

Beside the parabolic equations above, a linear model was also employed to construct the relationship between $u_{l}$ and $u_{n}$ of all ground motions set. Accordingly, the best fit linear equations for predicting $u_{n 50}$ and $u_{n 90}$ are:

$u_{n 50}=0.8522 u_{l}+0.06927$,

$u_{n 90}=0.9511+0.1002$.

The R-squared of these fitted equations are 0.99590 and 0.99649 for $u_{n 50}$ and $u_{n 90}$, respectively. 


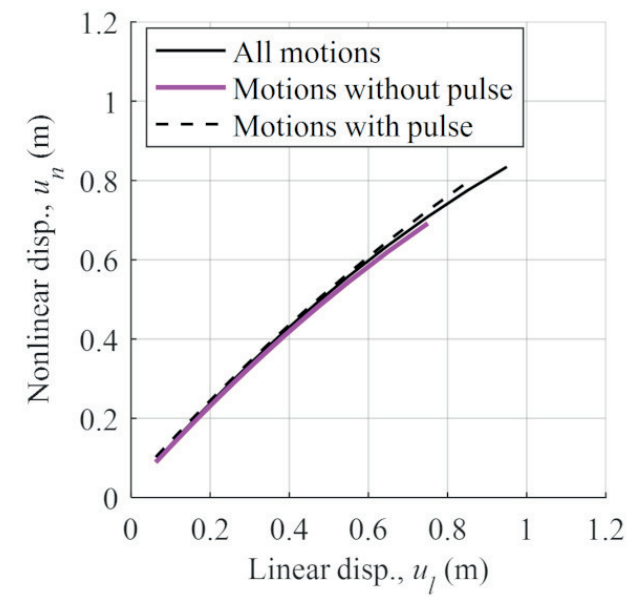

(a)

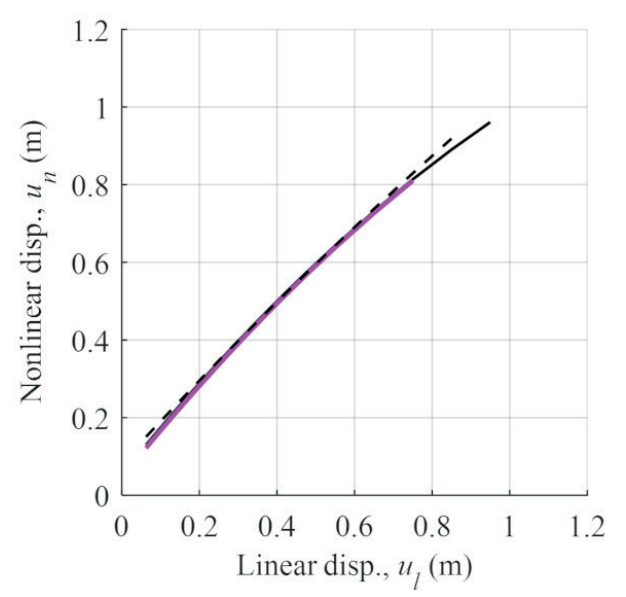

(b)

Fig. 12 Best fit curves for different ground motion sets a) Mean, b) 90 percentage

The linear fitting lines are plotted along with the parabolic fitting curves in Fig. 13. It can be observed that the linear lines are generally in good agreement with the corresponding parabolic curves. To provide a closer look at the accuracy of the linear fitting equations, the error between the linear equations and the data as well as the parabolic equations was computed and plotted in Fig. 14. The error between the parabolic equations and the data was also presented in the figure. As expected, the linear equations generate larger error than the parabolic equations, which produce very small error. As a result, the error between the linear equations and the data is close to the error between the linear equations and the parabolic equations. At a small $u_{l}$, the linear equations predict $u_{n 50}$ and $u_{n 90}$ with an error up to around $30 \%$ to the conservative side. At medium $u_{l}$, the linear equations slightly underestimate $u_{n 50}$ and $u_{n 90}$, with an error of less than $5 \%$. At very large displacement, the linear equations slightly overestimate $u_{n 50}$ and $u_{n 90}$.

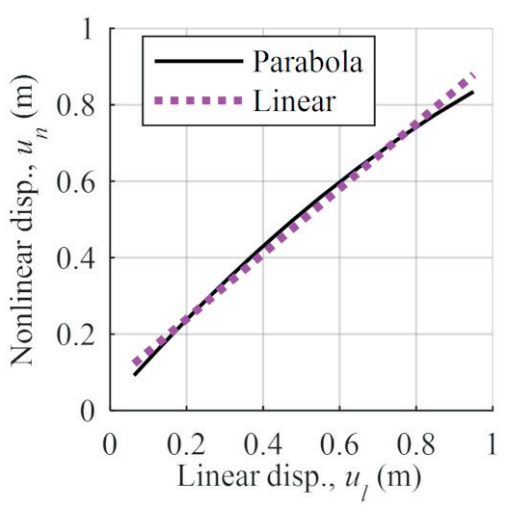

(a)

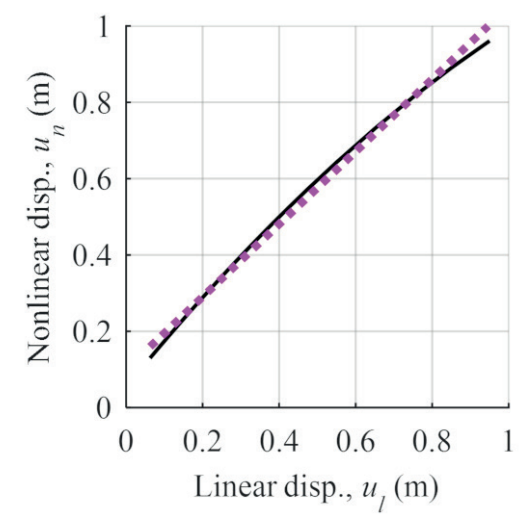

(b)

Fig. 13 Linear vs. parabolic fittings

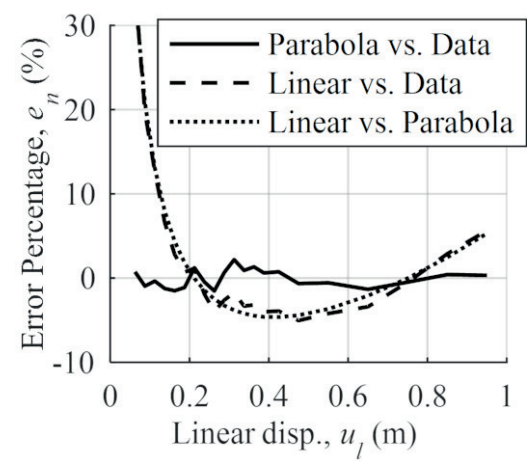

(a)

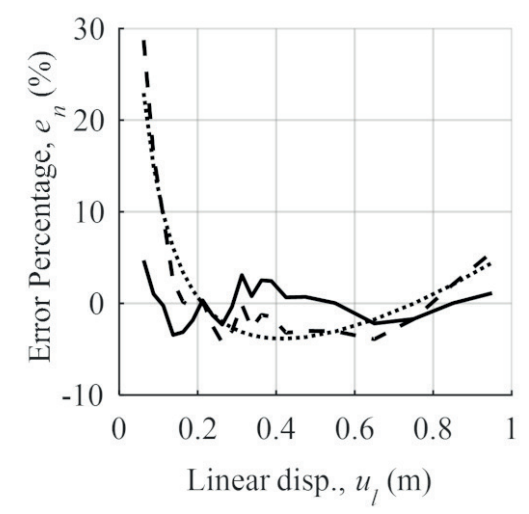

(b)

Fig. 14 Error percentage of prediction models 


\section{Conclusions}

This study evaluates and develops statistical relationships between the peak displacement predicted by an equivalent linear model and the peak displacement predicted by a nonlinear model of isolation systems using single friction pendulum bearings. The isolated systems were modeled as single mass systems with bidirectional movement in plan. This simple model was widely used in literature. The nonlinear isolation systems were modeled by a friction pendulum element with a velocity-dependent friction coefficient model. The fiction coefficient of the investigated systems ranged from 0.02 to 0.16 and their pendulum period ranged from $2.0 \mathrm{~s}$ to $5.5 \mathrm{~s}$. These wide ranges of parameters expect to cover most practical applications. The approximate linear model uses a secant stiffness at peak displacement and an equivalent linear viscous damping model following Jacobsen approach. Both nonlinear and equivalent linear systems were subjected to two earthquake ground motion types (i.e. motions without pulse and motions with pulse), represented by 390 strong pairs of recorded strong earthquake ground motion.

The peak displacements, which were obtained from dynamic time-history analysis of nonlinear and linearized models subjected to the selected ground motions, were statistically analyzed and the relationships between linear and nonlinear peak displacements at different reliability levels were developed. The results revealed that, on average sense, the equivalent linear model underestimates

\section{References}

[1] Naeim, F., Kelly, J. M. "Design of Seismic Isolated Structures: From Theory to Practice", Wiley, New York, NY, USA, 1999.

[2] ASCE "ASCE 7-16 Minimum design loads and associated criteria for buildings and other structures", American Society of Civil Engineers, Reston, VA, USA, 2017.

https://doi.org/10.1061/9780784414248

[3] CEN "BS EN 1998-5:2004 Eurocode 8: Design of structures for earthquake resistance", European Committee for Standardization, Brussels, Belgium, 2004.

[4] Guyader, A. C., Iwan, W. D. "Determining Equivalent Linear Parameters for Use in a Capacity Spectrum Method of Analysis", Journal of Structural Engineering, 132(1), pp. 59-67, 2006. https://doi.org/10.1061/(ASCE)0733-9445(2006)132:1(59)

[5] Caughey, T. K. "Equivalent linearization techniques", The Journal of the Acoustical Society of America, 35(11), pp. 1706-1711, 1963.

[6] Rosenblueth, E., Herrera, I. "Erratum for "On a Kind of Hysteretic Damping"', Journal of Engineering Mechanics Division, 91(3), pp. $37-48,1964$.

https://doi.org/10.1061/JMCEA3.0000643

[7] Franchin, P., Monti, G., Pinto, P. E. "On the accuracy of simplified methods for the analysis of isolated bridges", Earthquake Engineering and Structural Dynamics, 30, pp. 363-382, 2001. peak displacement at small displacement and overestimate it at large displacement. It is also observed that the relationship between linear and nonlinear peak displacement depends on ground motion types. Specifically, the relationships produce larger nonlinear peak displacement for motions with pulse and smaller nonlinear peak displacement for motions without pulse, given the same linear peak displacement.

The study constructed both parabolic and linear equations represented the relationship between linear and nonlinear peak displacement at different probability, namely $50 \%$ and $90 \%$, of exceedance. The relationship at $50 \%$ probability of exceedance predicts the expected value of nonlinear peak displacement given a linear peak displacement while the relationship at $90 \%$ probability of exceedance should be used for safety reason. As expected, the parabolic equations produce smaller error compared to the data than the linear equations. However, in practice, the linear equations can be used due to its simple form and its conservative error at small displacement.

Note that the results of this study are subjected to the limitations mentioned at the beginning of this section. Further studies should expand the investigation to $3 \mathrm{D}$ models to account for the flexibility of superstructures. Vertical component of ground motions should also be included. Besides, effect of friction coefficient models on the accuracy of the equivalent linear approach should also be considered.

[8] Dicleli, M., Buddaram, S. "Comprehensive evaluation of equivalent linear analysis method for seismic-isolated structures represented by SDOF systems", Engineering Structures, 29(8), pp. 1653-1663, 2007. https://doi.org/10.1016/j.engstruct.2006.09.013

[9] Mavronicola, E., Komodromos, P. "Assessing the suitability of equivalent linear elastic analysis of seismically isolated multi-storey buildings", Computer and Structures, 89(21-22), pp. 1920-1931, 2011.

https://doi.org/10.1016/j.compstruc.2011.05.010

[10] Jara, M., Jara, J. M., Olmos, B. A., Casas, J. R. "Improved procedure for equivalent linearization of bridges supported on hysteretic isolators", Engineering Structures, 35(2), pp. 99-106, 2012.

https://doi.org/10.1016/j.engstruct.2011.10.028

[11] Liu, T., Zordan, T., Briseghella, B., Zhang, Q. "Evaluation of equivalent linearization analysis methods for seismically isolated buildings characterized by SDOF systems", Engineering Structures, 59(2), pp. 619-634, 2014.

https://doi.org/10.1016/j.engstruct.2013.11.028

[12] Jara, J. M., Raya, G., Olmos, B. A., Martinez, G. “Applicability of equivalent linearization methods to irregular isolated bridges", Engineering Structures, 141, pp. 495-511, 2017. https://doi.org/10.1016/j.engstruct.2017.03.052 
[13] Dao, N. D., Nguyen-Van, H., Nguyen, T. H., Chung, A. B. "A new statistical equation for predicting nonlinear time history displacement of seismic isolation systems", Structures, 24, pp. 177-190, 2020.

https://doi.org/10.1016/j.istruc.2020.01.019

[14] Iwan, W. D., Gates, N. C. "The effective period and damping of a class of hysteretic structures", Earthquake Engineering and Structural Dynamics, 7(3), pp. 199-211, 1979. https://doi.org/10.1002/eqe.4290070302

[15] Miranda, E., Ruiz-García, J. "Evaluation of approximate methods to estimate maximum inelastic displacement demands", Earthquake Engineering and Structural Dynamics, 31(3), pp. 539-560, 2002. https://doi.org/10.1002/eqe.143

[16] Kwan, W.-P., Billington, S. L. "Influence of hysteretic behavior on equivalent period and damping of structural systems", Journal of Structural Engineering, 129, pp. 576-585, 2003. https://doi.org/10.1061/(ASCE)0733-9445(2003)129:5(576)

[17] Lin, Y.-Y., Miranda, E. "Evaluation of equivalent linear methods for estimating target displacements of existing structures", Engineering Structures, 31(12), pp. 3080-3089, 2009. https://doi.org/10.1016/j.engstruct.2009.08.009

[18] Makris, N., Kampas, G. "Estimating the "effective period" of bilinear systems with linearization methods, wavelet and time-domain analyses: From inelastic displacements to modal identification", Soil Dynamics and Earthquake Engineering, 45, pp. 80-88, 2013. https://doi.org/10.1016/j.soildyn.2012.11.007

[19] Papagiannopoulos, G. A. "Jacobsen's equivalent damping concept revisited", Soil Dynamics and Earthquake Engineering, 115, pp. 82-89, 2018.

https://doi.org/10.1016/j.soildyn.2018.08.001

[20] Rahgozar, N., Rahgozar, N., Moghadam, A. S "Equivalent linear model for fully self-centering earthquake-resisting systems", The Structural Design of Tall and Special Buildings, 28(1), Article ID e1565, 2019.

https://doi.org/10.1002/tal.1565

[21] Fadi, F., Constantinou, M. C. "Evaluation of simplified methods of analysis for structures with triple friction pendulum isolators", Earthquake Engineering and Structural Dynamics, 39, pp. 5-22, 2010.

https://doi.org/10.1002/eqe.930

[22] Morgan, T. A., Mahin, S. A. "The use of innovative base isolation systems to achieve complex seismic performance objectives", Pacific Earthquake Engineering Research Center, University of California, Berkeley, CA, USA, Rep. No. PEER-2011/06, 2011.

[23] Mokha, A., Constantinou, M., Reinhorn, A. M. "Teflon Bearings in Base Isolation I: Testing", Journal of Structural Engineering, 116(2), pp. 438-454, 1990. https://doi.org/10.1061/(ASCE)0733-9445(1990)116:2(438)

[24] Dolce, M., Cardone, D., Croatto, F. "Frictional Behavior of SteelPTFE Interfaces for Seismic Isolation", Bulletin of Earthquake Engineering, 3, pp. 75-99, 2005.

https://doi.org/10.1007/s10518-005-0187-9
[25] Quaglini, V., Dubini, P., Poggi, C. "Experimental assessment of sliding materials for seismic isolation systems", Bulletin of Earthquake Engineering, 10, pp. 717-740, 2012.

https://doi.org/10.1007/s10518-011-9308-9

[26] Fenz, D. M., Constantinou, M. C. "Modeling Triple Friction Pendulum Bearings for Response-History Analysis", Earthquake Spectra, 24(4), pp. 1011-1028, 2008. https://doi.org/10.1193/1.2982531

[27] Rabiei, M., Khoshnoudian, F. "Response of multistory friction pendulum base-isolated buildings including the vertical component of earthquakes", Canadian Journal of Civil Engineering, 38, pp. 10451059, 2011.

https://doi.org/10.1139/111-064

[28] Cardone, D., Gesualdi, G., Brancato, P. "Restoring capability of friction pendulum seismic isolation systems", Bulletin of Earthquake Engineering, 13(8), pp. 2449-2480, 2015. https://doi.org/10.1007/s10518-014-9719-5

[29] Ponzo, F. C., Cesare, A. D., Leccese, G., Nigro, D. "Shake table testing on restoring capability of double concave friction pendulum seismic isolation systems", Earthquake Engineering and Structural Dynamics, 46(14), pp. 2337-2353, 2017.

https://doi.org/10.1002/eqe.2907

[30] Constantinou, M., Mokha, A., Reinhorn, A. M. "Teflon Bearings in Base Isolation II: Modeling", Journal of Structural Engineering, 116(2), pp. 455-474, 1990. https://doi.org/10.1061/(ASCE)0733-9445(1990)116:2(455)

[31] Jacobsen, L. S. "Damping in composite structures", Proceedings of the Second World Conference on Earthquake Engineering, 2, pp. 1029-1044, 1960.

[32] Chopra, A. K. "Dynamics of structures: theory and applications to earthquake engineering", Prentice Hall, Upper Saddle River, NJ, USA, 2015.

[33] Nhan, D. D., Ai, C. B. "A constant friction coefficient model for concave friction bearings", Journal of Science and Technology in Civil Engineering, 14(1), pp. 112-126, 2020. https://oi.org/10.31814/stce.nuce2020-14(1)-10

[34] McKenna, F. "Open source for earthquake engineering simulation", University of California, Berkeley, CA, USA, 1997.

[35] Dao, N. D., Ryan, K. L., Sato, E., Sasaki, T. "Predicting the displacement of triple pendulum ${ }^{\mathrm{TM}}$ bearings in a full-scale shaking experiment using a three-dimensional element", Earthquake Engineering \& Structural Dynamics, 42(11), pp. 1677-1695, 2013. https://doi.org/10.1002/eqe.2293

[36] NEHRP "Selecting and scaling earthquake ground motions for performing response-history analyses", National Institute of Standards and Technology, Gaithersburg, MD, USA, Rep. NIST GCR 11-917$15,2011$.

[37] Becker, T. C. "Advanced modeling of the performance of structures supported on triple friction pendulum bearings", $\mathrm{PhD}$ Dissertation, University of California, 2011. 